\title{
Pandemic COVID-19, the High-Reliability Organization (HRO), and the Ecology of Fear
}

Daved van Stralen, MD, FAAP, Thomas A. Mercer, RAdm, USN

\begin{abstract}
:
COVID-19 has changed the social, financial, and political environments for healthcare. Healthcare organizations have abruptly changed operations for a new environment due to pervasive threats to the organization, patients, and healthcare professionals. While the direct, infectious presence of COVID-19 as a threat would seem to cause the greatest stress to the healthcare system, perhaps the absence of the threat may cause more severe and wide-ranging problems. Ecological studies demonstrate a greater decrease in prey populations from the predator's absence due to an "ecology of fear." By analogy, organizations share these trait responses and can develop an organizational culture of stress or stress. HRO describes functional traits for effective operations in environments of severe threat.
\end{abstract}

\section{"The tendency to discuss stress and fear as responses to threat may have misled us to diminish people's feelings, reasoning, and behaviors when the threat is absent. When nearby and visible, we can discuss measures to avoid attack and how to reduce psychological effects.."}

\section{Introduction:}

The tendency to discuss stress and fear as responses to threat may have misled us to diminish people's feelings, reasoning, and behaviors when the threat is absent. When nearby and visible, we can discuss measures to avoid attack and how to reduce psychological effects. The absence of threat, however, creates a pervasive unease that requires a response. The functional stress responses, fear reactions, and threat reflexes (1) work well to direct reason and action toward the threat. Without a target, however, these behaviors become misdirected and maladaptive.

Predators reduce prey populations to a greater extent by the absence of the predator rather than direct predation. The "ecology of fear" describes predator-prey interactions in the absence of the predator (2). Not only do prey populations decrease, but the ensuing trophic cascade changes the landscape to become a "landscape of fear" $(3,4)$.
COVID-19 has changed the social, financial, and political environments for healthcare, creating a new landscape. Healthcare organizations have abruptly changed operations for this new environment. While the direct, infectious presence of COVID-19 as a threat seems the greatest challenge, perhaps the absence of the threat may cause more severe and wide-ranging problems.

This paper will translate the ecology of fear through analogy to understand $\mathrm{HRO}$ as a culture for fear. By their absence, threats create an environment containing fear that interferes with operations. $\mathrm{HRO}$, as a verb, actively responds to the waxing and waning of threats while continuing operations in a normal manner. This combination of the environment with threat and continuous actions in response to the threat forms a culture that resists the stresses and fears or succumbs. HRO describes the functions not only to resist but to grow.
"This paper will translate the ecology of fear through analogy to understand HRO as a culture for fear. By their absence, threats create an environment containing fear that interferes with operations. HRO, as a verb, actively responds to the waxing and waning of threats while continuing operations in a normal manner."

\section{The Ecology of Fear:}

The direct killing of prey by a predator may have less influence on prey populations, and even the landscape, than the fear generated by the absence of a predator $(2,5)$. In the past two decades, fear has become a measurable element of ecology (6). By analogy, the fear of failure, in the absence of failure or the threat itself, may have a greater influence on human behavior and culture than actual failure (7). We will discuss this by analogy with mammalian ecosystems because of similar structures in predatorenemy-threat, prey-employees-organizations, and environmental stochasticity.

The fear of large carnivores changes the behavior of smaller carnivores and herbivores, which in turn alters the vegetation and landscape. For example, impala avoid woody areas due to predation by large carnivores. Experimental thinning of vegetation on the African savanna creates a safer environment that impala will enter

NEONATOLOGY TODAY is interested in publishing manuscripts from Neonatologists, Fellows, NNPs and those involved in caring for neonates on case studies, research results, hospital news, meeting announcements, and other pertinent topics.

Please submit your manuscript to: LomaLindaPublishingCompany@gmail.com 
to consume preferred, less-thorny Acacia trees. With the loss of competition, the thornier Acacia trees then become predominant. Grazing by larger herbivores, such as elephants, giraffes, eland, and oryx, that are not nearly as threatened by large carnivores, did not contribute to this change (8). The reintroduction of wolves in Yellowstone National Park changed the patterns of willow and cottonwood growth. Vegetation grew taller with significantly decreased growth of new vegetation that occurred where terrain features limited visibility or impeded escape by elk (9). The ecology of fear does not rely solely on the death of prey or the physical presence of predators. Introducing the sound of a large carnivore predator (dog in this study) into the environment of a smaller carnivore (raccoon in this study), compared to a non-predator (harbor seal in this study), had a significant influence on raccoon intertidal prey (10).

\section{"We use the term "stress" to encompass the organism's response to demands greater than a routine that marshal functional behavioral, metabolic, and neurobiological capabilities."}

We use the term "stress" to encompass the organism's response to demands greater than a routine that marshal functional behavioral, metabolic, and neurobiological capabilities. The "ecology of fear" includes the "stress response" of the hypothalamic-pituitaryadrenal (HPA) axis that releases glucocorticosteroids into the bloodstream and "fear reactions" that maintain a safe distance from a predator or other threat (11). The utility of the neuroanatomic and functional attributes helps interpret field studies that measure stress response through HPA activation and assess fear reactions through vigilance and maintenance of the "flight distance," a measurable distance that maintains safety (12).

In the wild, there is no risk-free environment. The absence of a predator does not mean the absence of risk; the lack of a threat perceived by the prey does not mean the absence of threat. To the prey, predation risk and the associated costs of antipredator defenses are an activity cost (13), while novelty, uncertainty, and uncontrollability are the fundamental causes of stress $(14,15)$. Increased vigilance when threats are predictable and controllable decreases food intake. Stress responses when threats are unpredictable and uncontrollable release systemic glucocorticoids, increase vigilance, change feeding locations, and reduce fecundity $(2,5)$.

Post-traumatic stress disorder (PTSD), through the stress response of HPA activation and fear reactions of vigilance and behavior changes, can link prey stress from predation with human stress. Animals in laboratory studies demonstrate 'sustained psychological stress' comparable to PTSD (16). However, various prey species in the wild have different HPA activation responses to persistent high predation risk. There is no HPA activation when lemmings or voles interact with weasels or when elk interact with wolves. There is, however, HPA activation when snowshoe hares or ground squirrels interact with predators (17).

On the other hand, wild animals develop persistent fear reactions similar to those observed in laboratory animals. Both groups demonstrate enduring effects on behavior such as hypervigilance and time spent in immobility and vigilance. Neuronal activation as enduring or immediate effects also meets the criteria for the animal model of PTSD (18). Predator exposure can lead to behavioral change that interferes with foraging over weeks to months (19).

\section{Fear of Disease:}

The ecology of fear can also develop from disease. The perception of predators elicits fear reactions for self-protection and to maintain a safe distance. Pathogens, on the other hand, elicit disease avoidance through disgust and fear, preventing contact with contagious objects (20). For example, disease-relevant invertebrates elicit greater fear and disgust relative to disease-irrelevant invertebrates (21).

Disgust for pathogen avoidance appears to be a conserved survival behavior. In tadpoles, chemical and/or vibrational cues released from cercariae induce hyperactivity with a movement away from the source (22). In their review, Jason Rohr, et al. (22) offered: tree frogs laying eggs use chemosensory detection to avoid pools containing trematode-infected snails, rainbow trout avoid cataract-causing trematode cercariae, and some vertebrates use defecation and foraging strategies to reduce fecal-oral parasitic

Table 1: Stress conditions

\begin{tabular}{|l|l|l|l|l|}
\hline Condition & Cause & Characteristics & Effects & Resolution \\
\hline Stress & $\begin{array}{l}\text { Novelty } \\
\text { Uncertainty } \\
\text { Uncontrollability }\end{array}$ & $\begin{array}{l}\text { Objective } \\
\text { Neurochemical } \\
\text { release }\end{array}$ & $\begin{array}{l}\text { Impaired declarative \& } \\
\text { working memory } \\
\text { Impaired cognition }\end{array}$ & Perception of control \\
\hline Fear & Proximity & $\begin{array}{l}\text { Subjective } \\
\text { Feeling }\end{array}$ & Maintain distance & Reframe \\
\hline Threat & Existential harm & $\begin{array}{l}\text { Objective } \\
\text { Behaviors }\end{array}$ & $\begin{array}{l}\text { Fight, anger } \\
\text { Flight, avoid } \\
\text { Freeze, vigilance } \\
\text { Tonic immobility, nausea } \\
\text { Dissociation }\end{array}$ & Conditioning \\
\hline
\end{tabular}


infection.

\section{Human Stress-Fear-Threat Cascade in an Ecology of Fear}

We will present fear ecology by analogy using the close alignment of the ecology of fear and the stress-fear-threat cascade (11). The functions of stress in the ecology of fear are listed in Table 1.From (11)

\section{"We can discuss fear ecology by analogy with mammalian ecosystems due to similar predator-enemy-threat, prey-employees-organizations, and environmental stochasticity. As in mammalian ecologies of fear, the stress HPA system is activated, vigilance increases vigilance, and behaviors change toward self-protection."}

We can discuss fear ecology by analogy with mammalian ecosystems due to similar predator-enemy-threat, prey-employeesorganizations, and environmental stochasticity. As in mammalian ecologies of fear, the stress HPA system is activated, vigilance increases vigilance, and behaviors change toward self-protection. In more constrained human societies (compared to wild animal communities), the ecology of fear initiates the stress-fear-threat cascade manifested through distinct human behaviors (11). In the $\mathrm{HRO}$, human performance strengthens the cascade's functions, while in the non-HRO, an ecology of fear leads to a rapidly malfunctioning cascade and performance deterioration. The manifestations of stress are listed in Table 2.

The fear of failure, rather than failure itself, itself, may have a greater influence on human behavior and culture than actual failure (7). We can correct or recover from failure, but we cannot cor- rect a fear. We will discuss this by analogy with mammalian ecosystems because of similar structures in predator-enemy-threat, prey-employees-organizations, and environmental stochasticity. The ecology of fear activates the stress HPA system, increases vigilance, and changes behaviors toward self-protection.

Paraphrasing a previous paragraph, stress is a property of the individual rather than a property of the threat. An HRO environment is not a risk-free environment. The absence of a threat does not mean the absence of risk. Hazard and threat-risk and the associated costs of reliability and safety defenses are an activity cost accepted by individuals and the organization. Safety behaviors and programs focus on unrecognized threats and hazards, the more insidious harm to operations. Novelty, uncertainty, and uncontrollability are the fundamental causes of stress. Individuals accept the duty, and organizations develop the structure for vigilance for and response to discrepancies and disruptions.,

We focus in this paper on stress responses and fear reactions, pervasive in the ecology of fear. We defer discussion of threat reflexes, which are an immediate response to an existential threat.

Stress responses functionally narrow cognition for focus on threats from abrupt contingencies and happenstance. However, even with mild stress, the amygdala will impair the executive functions and the prefrontal cortex (23). Increased stress response strength further impairs the prefrontal cortex and judgment, but imperceptibly so since we use our judgment to judge our judgment. The stress hormone cortisol impairs memory retrieval, interfering with cognitive capacity and memory consolidation, impeding allostatic learning. While close colleagues can recognize this in real-time, all participants become affected in the ecology of fear, decreasing the quality of collective judgment. Unrecognized threats and hazards will unpredictably, and likely unnoticed, damage operations.

Fear reactions functionally maintain a safe distance from the threat, giving a better perspective while also reducing the likelihood of existential harm. The individual can achieve distance from threat through diminishment, offensive protection, and defensive protection (11). The diminishment of the concerns of others about the threat increases the cognitive distance from the threat. More insidious is to question, devalue information, or impede information flow (24).

Table 2: Manifestations of stress conditions

\begin{tabular}{|l|l|l|l|}
\hline Condition & \multicolumn{1}{|c|}{ Mediator } & \multicolumn{1}{c|}{ Neurological Action } & \multicolumn{1}{c|}{ Manifestation } \\
\hline Stress & Amygdala & $\begin{array}{l}\text { Impaired prefrontal cortex } \\
\text { Impaired executive functions }\end{array}$ & $\begin{array}{l}\text { Loss of cognition } \\
\text { Subjectively rational } \\
\text { Objectively irrational; }\end{array}$ \\
\cline { 2 - 4 } & Cortisol & Impaired memory retrieval & $\begin{array}{l}\text { Confusion } \\
\text { Blunted recall }\end{array}$ \\
\hline Fear & $\begin{array}{l}\text { Ventromedial Prefron- } \\
\text { tal cortex } \\
\text { Periaqueductal Gray }\end{array}$ & Self-defense & $\begin{array}{l}\text { Move to safety } \\
\text { Offensive actions } \\
\text { Defensive actions }\end{array}$ \\
\hline Threat & Amygdala & Self-protection & $\begin{array}{l}\text { Anger, frustration } \\
\text { Plausible avoidance } \\
\text { Attentive freeze } \\
\text { Impeded decision-making }\end{array}$ \\
\hline
\end{tabular}


Offensive protection prompts aggressive attacks to stop the spread of the problem. The person will use surprise, concentrated actions, fast tempo, and audacity to achieve the feeling of security or control. Blame, accusation, and personal attacks are standard methods.

Defensive protection focuses on the individual's safety, often moving to a place of psychological or physical safety (25). Demands clearly exceed capabilities, and risks become too great for them to feel they can continue or survive. The person will not go near the threat or its source, whether abstract, such as concepts or specific information, or concrete, such as the leader, an administrator, or a colleague. Because the individual will not sufficiently approach the situation, descriptions, correlations, or causations do not develop. As a result, individuals must rely on rationalizations and abstractions (for example, clichés and metaphors) to support and explain judgments, interpretations, and actions. The individual is less useful to protect others since the person becomes focused primarily to reduce risk to themselves. Deflection, excuses, justifications, and prophylactic self-blame are standard methods.

\section{"The person will not go near the threat or its source, whether abstract, such as concepts or specific information, or concrete, such as the leader, an administrator, or a colleague. Because the individual will not sufficiently approach the situation, descriptions, correlations, or causations do not develop."}

Embedded in fear reactions in the ecology of human and organizational fear are offensive and defensive acts to protect the person's ego. The unrecognized threat to ego is possibly the most dangerous situation in the ecology of fear.

\section{A Virus Ecology of Fear:}

A pandemic virus-as-predator might create an ecology of fear within a society, observed Raymond Novaco, University of California, Irvine (personal communication).

\section{Ebola Virus Disease (EVD)}

Gregg Mitman (26) described the fear surrounding the EVD as having its own ecology that needed to be understood. The EVD epidemic demonstrated the effects of the ecology of fear when fear of a disease combines with prejudice (US) and painful history (Africa). Western attitudes associate equatorial Africa with deadly tropical diseases, generating irrational fears about the pathogenesis and infectious nature of the Ebola virus, an RNA virus $(26,27)$.

Incomplete information and historical distrust in authorities, particularly western authorities, contribute to rumors that will naturally emerge during an evolving event. Constrained health literacy contributes to resistance against effective healthcare principles. People in western countries then dismiss reasoned rumors and well-founded resistance as simple ignorance and superstition (28). Some US school officials and college admissions programs banned students or rejected applicants from African nations, even as far as 1,700 miles from the exposed region (27).

The social fabric expected to hold society together began to tear. Caring for the sick and dead within Sierra Leone, Liberia, and Guinea would bring sickness and death (28). Fears stigmatized survivors. In the US, shunning behavior was directed against healthcare providers close to hospitalized quarantined patients (27).

The fear of creating an EVD fear ecology may have originated from the fear of an unknown infection in a land with a long history of exploitation by western nations (26-28).

Suspicion of healthcare workers and response teams was less a rejection of western medicine than re-experiencing "sub-standard and heavy-handed provision" of care in the past (27). The EVD epidemic occurred in an area with a history of "European and West African slave traders, white missionaries, and Liberian soldiers recently sent to conquer the interior" and painful memories of past medical encounters in West Africa (26). This history and exploitation by western countries contributed to fear and conspiracy theories (27).

The severity of EVD, with limited knowledge about transmissibility and effective treatment, made EVD an accessible vehicle for politics, with response teams becoming deeply politicized (28). The consequences of politicizing fear and prejudice in the historical background of abuse and marginalization lead to violent anger. The consequent Ebola ecology of fear may explain violent fatal attacks on healthcare providers and volunteers $(27,28)$.

\section{COVID-19}

A previously unidentified coronavirus, also an RNA virus from a geographic and cultural region dissimilar to the US, led to a worldwide pandemic. The social response to COVID-19 is quite similar to the response to EVD, though on a larger, more pervasive, and intense scale.

\section{"The consequences of politicizing fear and prejudice in the historical background of abuse and marginalization lead to violent anger."}

Though pathogens tend to elicit disease avoidance, the present human and social response to COVID-19 bears more semblance to the fear reactions elicited by predators. We can appreciate COVID-19 fear ecology through an analogy using the close alignment of the ecology of fear, the stress-fear-threat cascade (11), and the recent experience with EVD.

Ecology of fear and the recent experience with EVD describes some of our current problems with a virus that, as stated by Mitman (26), has its own ecology that needs to be understood. The current COVID-19 pandemic combines prejudice and painful history, complicates, and confounds our best efforts to achieve a successful resolution. Attitudes associated with "the other not like us" and incomplete information about an evolving disease generates 
irrational fears and further misinformation.

Incomplete information and distrust in authorities and scientific experts during an evolving event contribute to rumors that naturally emerge from confusion and uncertainty. People reduce the stress of novelty by anchoring information to what they believe-gaining a sense of autonomy, even if in conflict with prudent healthcare or public health principles, brings predictability and controllability. Finding the familiar in the novel, seeking certainty even if contrived, and controlling one's behaviors and beliefs will reduce the causes of stress - novelty, uncertainty, and uncontrollability (14, 15).

Constrained health literacy, almost an endemic problem, contributes to resistance against effective healthcare principles. Dismissing non-medical beliefs increases the resistance to prudent and effective public health measures.

The social fabric that we expect will hold society together has begun to tear as in West Africa during the EVD epidemic. Fears stigmatize those with whom we disagree. Shunning behavior becomes directed against healthcare providers and public health officials.

Suspicion of healthcare workers and response teams may be less a rejection of medical practice than re-experiencing impersonal, "sub-standard, and heavy-handed provision" of care by the healthcare community. In some communities and minority groups, painful memories of denied or limited medical care remain in the collective memory. One author (DvS) reminds healthcare staff before and after discussions for limitation or withdrawal of medical care for a child of a minority that a family member or friend likely had medical care denied or limited because of race. That experience will be at the forefront of their mind as we discuss limiting medical care for defined ethical reasons. This is personal to them rather than the larger issue of healthcare equity.

COVID-19 had a confusing presentation with a wide range of severity, the facility of its spread, and a misleading mortality rate. Combined with the initially limited knowledge about transmissibility and effective treatment, COVID-19 became an accessible vehicle for politics.

Viewing the COVID-19 or EVD responses as an ecology of fear reveals the similarities of the fear environment created by the viruses. Because the predator is not physically present, viruses can act like a predator. But an ecology of fear was likely already present. People can treat abstractions as a concrete rendering of a predator, allowing them to respond to abstractions like error, failure, liability, even someone's fear, as they would a predator. Alfred North Whitehead (29) warned against this "fallacy of misplaced concreteness," mistaking the abstract for the concrete.

\section{"Fears stigmatize those with whom we disagree. Shunning behavior becomes directed against healthcare providers and public health officials."}

When created from abstractions, the ecology of fear forms an environment with mutual threats, but the importance or degree of mutual threats is not equally shared. Error, failure, and liability as abstractions of mutual threat create or influence a fear environment, but the fear is not equally distributed. Employees, managers, supervisors, administrators, regulators, and executives also create a fear environment. Any abstraction considered a predator, along with the ecology of fear, creates a fear environment. Culture as the social response to the environment can effectively extend a group into a fear environment, or the culture can generate avoidance or withdrawal. $\mathrm{HRO}$, as a culture, extends the organization into an ecology of fear (30).

\section{Stress As A Culture:}

Cultures function within an environment and are continued through shared social knowledge. Stress and the ecology of fear can influence cultures toward homeostasis and protection against change or generate allostasis and strength through change. Stress can predominate in the organization's operational environment, or stress can become a form of acquired knowledge to interpret experience and generate behavior. We can describe cultures, regardless of the model used, with the same characteristics: an environment in which the culture developed, behaviors useful in the environment, and some are norms specific to a culture, beliefs as invariant values or adaptive attitudes, and some artifact or technology shaping the culture. Rules and procedures form the artifacts in many organizations.

Cultural ecology is the "ways in which culture change is induced by adaptation to the environment" (31). The environment, even the ecology of fear, influences human adaptation but does not determine adaptation. Steward's (31) primary arguments were that (1) cultures in similar environments might have similar adaptations; (2) all adaptations are short-lived and are continually adjusting to changing environments, and (3) changes in culture can elaborate on an existing culture or can result in the creation of entirely new ones.

Culture is the knowledge acquired and shared and how knowledge is used to interpret experience and generate behavior (32). As people learn their culture, they acquire new ways to interpret experience

Sources of social knowledge include social learning by observation of other people (33). These individuals may be found within the organization or during other social interactions. Social learning also occurs through movies and television. The author (DvS) noted a change in behavior from bystanders during fire rescue ambulance responses. People stood closer and watched rather than becoming involved and helping, questions became directed toward what paramedics should do rather than concern about the patient's condition, and expectations for specific actions and behaviors began to appear. Colleagues pointed the author to a television show, Emergency!, which showed these behaviors. Perhaps the realistic portrayal of physician, nurse, and paramedic relationships and interactions facilitated the transfer from what people watched on television to what they saw when paramedics were on scene. The realistic interactions were by design. Ronald Stewart, the medical advisor to the show, broke with the tradition of vetting physician roles by the AMA that maintains a physician's distance from nurses and ancillary staff. Stewart had designed paramedic education and operations in Los Angeles County to drive discussion between physicians, nurses, and paramedics. He wanted the realism of that approach in the show (personal com- 
munication).

Unfortunately, the social learning of some stress behaviors through movie and television representations has become common. Character-driven movies with introspective lead characters, such as the 1953 Western movie Shane where the gunfighter shows gentle, controlled strength, have been replaced with action, retribution, and vengeance movies. Maladaptive and dysfunctional responses to abrupt contingencies have become more acceptable and the norm in some areas. Early in medical school, one author (DvS) encountered the belief that to learn stress; one had to be placed under stress, "sink or swim." When queried for the source of the belief, it was how the military trained recruits. Because none of the respondents had military service, the next query was how one would know. The consistent answer, "It's in the movies." Hollywood clichés of emotional bonding in the military may also influence soldiers and social scientists (34) as well as healthcare professionals. Influenced by movies and television, the environment becomes distorted as unsafe while maladaptive behaviors become accepted that further distort the environment.

\section{"Social learning also occurs through movies and television. The author (DvS) noted a change in behavior from bystanders during fire rescue ambulance responses. People stood closer and watched rather than becoming involved and helping, questions became directed toward what paramedics should do rather than concern about the patient's condition, and expectations for specific actions and behaviors began to appear."}

Stress and fear are attack vectors aimed at the individual or organization but can readily become attack vectors for organizational failure when error becomes identified as a threat (7). Organizations may use threat and stress responses for compliance and obedience and to support directive or authoritarian leadership styles. Paradoxically, while stress as a concept brings comprehension, it robs the individual of the sense of controllability. Stress, fear, and threat then become affective experiences, constructing an environment built from subjective stress and nearly independent of objective threats. That is, rather than engaging in a situation, individuals evaluate threat as risk, judging whether to engage. Threat and stress become the environment and ecology of fear. For example, faced with an explosive device or a burning train inside a tunnel, a prudent individual might weigh the risk and benefits of rescue. Yet, when queried about civilians trapped near an explosive device, a San Bernardino City (California) Police Sergeant, with that experience, immediately remarked the officers would continue extrication (as they had done with some criticism from outside), "We won't leave someone alone." When asked why the fire rescue team would approach a burning train within a tunnel, a French Division Chief, Bouches du Rhone Fire Department
(France), answered, "The French people expect us to come to their aid." Yet, in healthcare, concern for liability informs the working environment and the possibility of "doing harm" informs the practice environment.

Stress as a functional property of the individual $(1,11)$, rather than a property of the predator or threat, allows us to discuss stress responses, fear reactions, and threat reflexes as situational functions. Rather than missed as part of the environment, stress becomes visible as acquired social knowledge, contributing to the maintenance of culture. Stress, fear, and threat are knowledge: the salience and meaning of stimuli, the interpretation of events, information flow and communication, and interactions between leader and subordinate. To evaluate stress as social knowledge, we ask, "How do people in the culture use or respond to stress?" The HRO uses threat and stress responses as drivers to increase capability toward engagement. Others may use threat and stress responses to avoid a threat, reduce risk, or enforce compliance.

We can now see culture in terms of interactions with a small-scale environment, a process operating as the social group continually adapts to the environment (31). This "cultural ecology" represents the "ways in which culture change is induced by adaptation to the environment" (31). Threat or a predator, as an independent component of the environment, then informs the cultural response of stress and fear and the cultural response becomes social knowledge shared with members of the organization. The characteristics of an HRO begin to degrade.

The HRO uses threat and stress responses as drivers to learn, inform leadership styles $(35,36)$, team formation, and foster collegiality. As acquired cultural knowledge, stress informs the detection and response to threats and identifies and modulates stress responses. Rather than forming around stress when engaging adversity or entering an adverse environment, the HRO leverages stress into safety and reliability (7).

A culture of stress debilitates people and weakens organizational responses. A culture for stress strengthens the individual. It is the individual who believes in themselves who decides to move forward and engage. HRO is a culture for stress.

\section{Organizational Response To Fear:}

Nobel laureate Niko Tinbergen, writing "Every animal has to cope in numerous ways with a hostile, at least uncooperative environment," posited that it is behavior that gives the animal the ability to cope and survive (35). Measurable antipredator behaviors in the ecology of fear when the predator is absent include vigilance, sociality, location, and feeding (when and where). Decision-making by prey includes trade-offs between the risk of predation, the benefits of the activity, and the ability for antipredator behavior $(5,13)$. Patterns of defenses differ if the risk is unpredictable, uncontrollable, variable, and defense costs are high (5).

Proactive defenses have the greatest effectiveness when risks are predictable and controllable. Reactive defenses are more effective and reliable with increasingly unpredictable or uncontrollable risk. When risks are consistently high or if defensive costs are low, then fixed constitutive defenses, such as spines, become effective. More often, risks will vary by location or over time, and defenses carry costs (5). Vigilance in the absence of the predator, a defense cost, sustains the stress response with chronically elevated glucocorticoid levels and reduced reproduction $(2,16)$. 
Inducible antipredator responses allow the selection of antipredator behaviors with variable expression, increased behaviors for elevated risks, and decreased expression as the risk abates (5).

In organizational terms, proactive, fixed constitutive defenses make sense for their effectiveness and lower cost, becoming a normative stance from an external, fixed reference frame. On the other hand, the pragmatic frame corresponds to the adaptive expression of behaviors selected by the individual at the point of contact (30). A fundamental problem lies in the interpretation of error. During a risky episode, behaviors will likely deviate from rules. From the normative stance, these deviations signal the possibility of error, while from the pragmatic stance, these deviations may be considered an error, though the pragmatist sees adaptability and finds utility in the information generated. Notions of high reliability make a big deal of this difference. Control operators, like those in a nuclear power facility, prevent failure. Deviation from rules signals potential failure. Like wildland firefighters, emergency responders respond to failure, and tight adherence to rules signals potential failure (36).

\section{"A culture of stress debilitates people and weakens organizational responses. A culture for stress strengthens the individual. It is the individual who believes in themselves who decides to move forward and engage. HRO is a culture for stress."}

Adaptive expression of behaviors for defense also performs well with competing objectives, where one objective appears to threaten another objective. Neonatologists work toward goals with varying time horizons generating varied temporal perspectives: how does resuscitation affect maternal bonding, social development of the family, and organ development after NICU discharge? Neonatologists routinely work with multiple, conflicting objectives, varying timelines, and limited and imperfect information. Despite this variability, neonatologists share a drive to achieve the same end-state - bonding of infant and family in the best possible physiological condition. For example, a review of multiple national COVID-19 treatment guidelines from various nations showed similarities in treatment. All national groups, however, produced their guidelines for the same, shared end-state (37). Variability creates stability in an uncertain world.

This notion that behavior contributes to failure by way of error leads organizations to constrain adaptive behaviors increasingly during a crisis. The mistranslation of error from a method to correct heuristic bias (38) and identify the limits of operations and performance (the "performance" or "operational envelope") has turned an error into an early herald of failure (7). But it is the contextual expression of behaviors, utilizing reciprocal feedback, and correcting errors that generate improvisation (39), even under lifethreat (40). Perhaps the gap between the different logics of theory and practice (41) informs this mistranslation. The scientific logic for theory creates mental objects, abstractions not "found" through inquiry but inference (42). In an ecology of fear, we cannot accept these inferred abstractions as concrete representations (29). Representational logic underlies scientific theory while the operator is immersed in the ecology of fear where entities and events continuously and constantly change. Operators in this engaged practice use practical rationality to form a logic of practice, a logic that is not well worked out (43).

Following a year engaged in a "behavior versus concepts" controversy with experts and specialists from multiple industries, the author (DvS) received the following note of encouragement from Karl Weick: "John Dewey agrees with your emphasis on behavior" followed by this quotation:

John Dewey insisted that inquiry is always a behavioral response of a reflective organism to its environing conditions....inquiry belongs to "action or behavior, which takes place in the world, not just within the mind or within consciousness.... Inquiry, just as much as walking or eating, is what Dewey termed an "outdoor fact." (42).

Defensive measures protect the organization from damage due to direct attack and also protect routine operations from distractions. Karl Weick (personal communication) once described how his motivation for "sensitivity to operations" came from studies that demonstrated failure when a disruption had distracted the organization from their routine operations. It would appear prudent to cancel or defer risky studies that do not have an immediate benefit for a neonate in the NICU. Risks, however, may have a window of treatment before irreversible damage occurs. For example, the mechanism of damage to incompletely developed organs is similar to Acute Radiation Syndrome (ARS): the pathogen is hidden (ionizing radiation/cellular damage from hypoxia), the damage is delayed, and the disease is untreatable. The problem of fissile material causing ARS contributed directly to the development of safety culture in the nuclear power industry (44). Retinal cells of the premature neonate provide several examples. Drugs that may damage a small percentage of vision cells may cost an adult only a small percentage of cells with limited effect on vision. For a premature infant, those damaged cells represent a logarithmic growth of vision. For Retinopathy of Prematurity, Candace Frazier (45) identified methods for monitoring ROP that limit exposure to COVID-19 through membranes around the eye through the use of telemedicine and ultra-widefield imaging.

In the ecology of fear, the influence of the predator is through its absence. For an HRO, the influences of threats are through their absence, less of a measurable probability and more of the ease, or possibility, for abrupt change. The five characteristics of HRO (46) initiate action against threat or adversity. Preoccupation with failure describes vigilance toward absent threats; reluctance to simplify acknowledges the latent presence of threats before they become visible; sensitivity to operations guarantees continued operations of the organization for logistic support to exigencies; commitment to resilience ensures engaging the problem, decomposing objectives as needed; and deference to expertise entrains resources into the engagement.

Organizations maintain vigilance for outliers in operational terms, treating them as early heralds of attack rather than random, independent events that can be disregarded. Engagement initiates sensemaking by generating information and creating structure through action (30). Enactment driven by the organization's cul- 
ture changes the environment from an ecology of fear and generates allostatic stability through change.

\section{"As a continuous process, doing in emergencies what is done every day, the organization responds to, recovers from and incorporates minor insults and major disruptions while moving forward. Rather than returning to the pre-crisis state, this form of resilience generates dynamic stability and strength through change."}

\section{Conclusion:}

Stress describes the organism's response to novelty, uncertainty, or uncontrollability $(14,15)$. Fear describes the response to the proximity of a threat $(12,47)$. We limit our discussion of threat reflexes reacting to existential threat $(48,49)$ because stress responses and fear reactions can be sustained in the absence of a threat $(16,18,19)$ while the direct, personal closeness of existential threat triggers threat reflexes that more quickly resolve.

The functional value of stress can become dysfunctional without adaptive neuromodulation (1). Functionally, stress describes the stress responses to constrain reason and thought fear reactions to keep the threat at a safe distance, and threat reflexes for existential survival (11). Stress responses can be "linked to mere thoughts" (50), and fear responses can be generated by the absence of a predator $(2,5)$.

In the larger, environmental context, stress and fear can shape an ecology of fear (2), which then informs human culture into a culture of fear or culture for fear. HRO describes the characteristics (46) and attributes $(30,38)$, allowing an organization to continue operations during severe disruptions or extend operations into a volatile, threatening environment.

Acknowledging the function of stress and the survival value of behaviors, the organization can produce distinct proactive, constitutive defenses for predictable and controllable risks, even for consistently high risks. Planning becomes focused and substantive. On the other hand, for unpredictable or uncontrollable risks, or risks that vary with time and place, reactive defenses may prove more effective and reliable. When defense carries costs, the organization can plan for actions expressed for situations heralding system dysfunctions, evoked by early heralds, acting before problems begin entraining resources.

An outlier becomes noticed as the beginning of unwanted processes for further inquiry rather than a random, independent event that can be readily disregarded. Error then becomes a tool, in effect an artifact that informs the organization's culture, a marker for the boundaries of the operational envelope, indicators for a changing environment, revealing novel or unexpected elements.

In an environment of disruption, an ecology of fear, the adaptive organization supports vigilance for early heralds of failure. Not preoccupied at the expense of other duties, but vigilance as sensitivity to operations, maintaining operations during disruption while also resolving the disruption. Engage what initially appears simple but through inquiry revealing more complex salient elements. The increased granularity brings out relevant, local expertise for more effective actions. As a continuous process, doing in emergencies what is done every day, the organization responds to, recovers from and incorporates minor insults and major disruptions while moving forward. Rather than returning to the pre-crisis state, this form of resilience generates dynamic stability and strength through change. This is HRO; rather than responding to the future, the organization prepares for the future through daily operations.

\section{References:}

1. Daved van Stralen TAM. Pragmatic High-Reliability Organizations (HRO) Modulate the Functions of Stress and Fear Behaviors during Pandemic COVID-19: The Stress-FearThreat Cascade. Neonatology Today. 2020;15(10):126-34.

2. Joel S. Brown JWL, and Mahesh Gurung. The Ecology of Fear: Optimal Foraging, Game Theory, and Trophic Interactions. Journal of Mammalogy. 1999;80(2):385-99.

3. Laundré JW, Lucina Hernández, and Kelly B. Altendorf. Wolves, elk, and bison: reestablishing the "landscape of fear" in Yellowstone National Park, USA. Canadian Journal of Zoology. 2001;79(8):1401-9.

4. Laundré JW, Lucina Hernández, and William J. Ripple. The landscape of fear: ecological implications of being afraid. The Open Ecology Journal 2010;3(1).

5. Creel S. The control of risk hypothesis: Reactive vs. proactive antipredator responses and stress-mediated vs. food-mediated costs of response. Ecology letters 2018;21(7):947-56.

6. Laundré JW, Lucina Hernández, and William J. The landscape of fear: ecological implications of being afraid. The Open Ecology Journal 2010;3(1):1-7.

7. Daved van Stralen WG. Error as a Faulty Failure Signal. Neonatology Today. 2020;15(8):114-7.

8. Ford AT, Jacob R. Goheen, Tobias O. Otieno, Laura Bidner, Lynne A. Isbell, Todd M. Palmer, David Ward, Rosie Woodroffe, and Robert M. Pringle. Large carnivores make savanna tree communities less thorny. Science 2014;346(6207):346-9.

9. Ripple WJ, and Robert L. Beschta. Wolf reintroduction, predation risk, and cottonwood recovery in Yellowstone National Park. Forest Ecology and Management 2003;184(1-3):299-313.

10. Suraci JP, Michael Clinchy, Lawrence M. Dill, Devin Roberts, and Liana Y. Zanette. Fear of large carnivores causes a trophic cascade. Nature communications 2016;7(1):1-7.

11. Daved van Stralen TAM. During Pandemic COVID-19, the High-Reliability Organizations (HRO) Identifies Maladaptive stress Behaviors: The Stress-Fear-Threat Cascade. Neonatology Today. 2020;15(11):113-23.

12. Hediger H. Wild Animals in Captivity London, UK Butterworths Scientific Publications; 1950.

13. Lima SL, and Lawrence M. Dill. Behavioral decisions made under the risk of predation: a review and prospectus. Canadian journal of zoology 1990;68(4):619-49.

14. Mason JW. A review of psychoendocrine research on the pituitary-adrenal cortical system. Psychosomatic medicine 1968;30(5):576-607.

15. Gagnon SA, and Anthony D. Wagner. Acute stress and episodic memory retrieval: neurobiological mechanisms and behavioral consequences. Annals of the New York Academy of Sciences. 2016;1369(1):55-75. 
16. Clinchy $M$, Sheriff MJ, Zanette LY, Boonstra R. Predatorinduced stress and the ecology of fear. Functional Ecology. 2013;27(1):56-65. doi: 10.1111/1365-2435.12007.

17. Boonstra R. Reality as the leading cause of stress: rethinking the impact of chronic stress in nature. Functional Ecology 2013;27(1):11-23.

18. Zanette LY, Emma C. Hobbs, Lauren E. Witterick, Scott A. MacDougall-Shackleton, and Michael Clinchy. Predatorinduced fear causes PTSD-like changes in the brains and behaviour of wild animals. Scientific reports 2019;9(1):1-10.

19. Zanette LY, Clinchy M. Ecology and Neurobiology of Fear in Free-Living Wildlife. Annual Review of Ecology, Evolution, and Systematics. 2020;51(1):297-318. doi: 10.1146/ annurev-ecolsys-011720-124613.

20. Prokop $P$, and Jana Fančovičová. Self-protection versus disease avoidance. Journal of Individual Differences. 2013;34:15-23.

21. Prokop P, Jana Fančovičová, and Peter Fedor. Health is associated with antiparasite behavior and fear of disease-relevant animals in humans. Ecological Psychology 2010;22(3):222-37.

22. Rohr JR, Swan A, Raffel TR, Hudson PJ. Parasites, info-disruption, and the ecology of fear. Oecologia. 2009;159(2):44754. Epub 2008/11/08. doi: 10.1007/s00442-008-1208-6. PubMed PMID: 18989706.

23. ArnstenAF. Stress signalling pathways that impair prefrontal cortex structure and function. Nat Rev Neurosci. 2009;10(6):41022. Epub 2009/05/21. doi: 10.1038/nrn2648. PubMed PMID: 19455173; PubMed Central PMCID: PMCPMC2907136.

24. Westrum R. A typology of organisational cultures. Quality and Safety in Health Care. 2004;13(suppl_2):ii22-ii7. doi: 10.1136/qshc.2003.009522.

25. Oatley K, Johnson-Laird PN. Cognitive approaches to emotions. Trends Cogn Sci. 2014;18(3):134-40. Epub 2014/01/07. doi: 10.1016/j.tics.2013.12.004. PubMed PMID: 24389368.

26. Mitman G. Ebola in a stew of fear. New England Journal of Medicine. 2014;371(19):1763-5.

27. Karamouzian M, Hategekimana C. Ebola treatment and prevention are not the only battles: understanding Ebola-related fear and stigma. Int $J$ Health Policy Manag. 2015;4(1):556. Epub 2015/01/15. doi: 10.15171/ijhpm.2014.128. PubMed PMID: 25584356; PubMed Central PMCID: PMCPMC4289040.

28. Wilkinson A, Leach M. Briefing: Ebola - Myths, Realities, and Structural Violence. Afr Affairs. 2015;114(454):13648. doi: 10.1093/afraf/adu080. PubMed PMID: WOS:000347808800007.

29. Whitehead AN. Science and the Modern World. Cambridge: Cambridge University Press; 1926.

30. Stralen Dv. Pragmatic HRO during Pandemic Covid-19. Neonatology Today. 2020;15(4):3-9.

31. Steward JH. Theory of Culture Change: The Methodology of Multilinear Evolution. Urbana,IL: University of Illinois Press; 1955.

32. Spradley JP. Culture and Ethnography. In: McCurdy JPSaDW, editor. Conformity and Conflict: Readings in Cultural Anthropology. Boston, MA: Pearson; 1984. p. 1-13.

33. Bandura A. Social Foundations of Thought and Action: A Social Cognitive Theory. Englewood Cliffs, NJ,: Prentice-Hall 1986.

34. MacCoun RJ, Elizabeth Kier, and Aaron Belkin. . Does social cohesion determine motivation in combat? An old question with an old answer. Armed Forces \& Society 2006;32(4):646-54.

35. Tinbergen N. On Aims and Methods of Ethology. Zeitschrift fur Tierpsychologie [Journal of comparative ethology]. 1963;20(4):410-33

36. Roe E, Schulman PR. Comparing Emergency Response Infrastructure to Other Critical Infrastructures in the California Bay-Delta of the United States: A Research Note on Inter-Infrastructural Differences in Reliability Management. Journal of Contingencies and Crisis Management. 2015;23(4):193200. doi: 10.1111/1468-5973.12083.

37. Ahmed Afifi DC, Allison C. Walker, Alexandra Adamczak, T. Allen Merritt, Jan Mazela, Thomas A. Clarke Guidelines for Management of Infants Delivered during the COVID-19 Pandemic in the USA and "Across the Pond" Neonatology Today. 2020;15(6):3-29.

38. Daved van Stralen SDM, Thomas A. Mercer. Flight Decks and Isolettes: High-Reliability Organizing (HRO) as Pragmatic Leadership Principles during Pandemic COVID-19. Neonatology Today. 2020;15(7):113-22.

39. Weick KE. Introductory Essay-Improvisation as a Mindset for Organizational Analysis. Organization Science. 1998;9(5):543-55. doi: 10.1287/orsc.9.5.543.

40. van Stralen D, Sean D. McKay, George T. Williams, Thomas A. Mercer. Tactical Improvisation: After-Action/ Comprehensive Analysis of the Active Shooter Incident Response by the San Bernardino City Fire Department December 2, 2015. San Bernardino, CA: 2017.

41. Zundel $M$, and Panagiotis Kokkalis. Theorizing as engaged practice. Organization Studies 2010;31(9-10):1209-27.

42. Hickman LA. Pragmatism as post-postmodernism: Lessons from John Dewey. New York, NY: Fordham University Press; 2007.

43. Sandberg J, and Haridimos Tsoukas. Grasping the Logic of Practice: Theorizing through Practicality Rationality. Academy of Management Review 2011;36(2):338-60.

44. Van Stralen D, Spencer Byrum and Bahadir Inozu. High Reliability for a Highly Unreliable World: Preparing for Code Blue through Daily Operations in Healthcare. North Charleston, SC: CreateSpace Publishing; 2017.

45. Frazier CD. COVID-19 Response: Safety for ROP Screening in Neonatal Intensive Care Unit. Neonatology Today. 2020;15(11):19-22.

46. Weick KE, and Kathleen M. Sutcliffe. Managing the Unexpected: Resilient Performance in a Complex World,. Hoboken, NJ: John Wiley \& Sons; 2015.

47. Mobbs D, Predrag Petrovic, Jennifer L. Marchant, Demis Hassabis, Nikolaus Weiskopf, Ben Seymour, Raymond J. Dolan, and Christopher D. Frith. When fear is near: threat imminence elicits prefrontal-periaqueductal gray shifts in humans. Science. 2007;317(5841):1079-83.

48. LeDoux JE, Pine DS. Using Neuroscience to Help Understand Fear and Anxiety: A Two-System Framework. Am J Psychiatry. 2016;173(11):1083-93. Epub 2016/11/02. doi: 10.1176/ appi.ajp.2016.16030353. PubMed PMID: 27609244.

49. LeDoux JE. Coming to terms with fear. Proc Natl Acad Sci U S A. 2014;111(8):2871-8. Epub 2014/02/07. doi: 10.1073/ pnas.1400335111. PubMed PMID: 24501122; PubMed Central PMCID: PMCPMC3939902.

50. Sapolsky RM. Why zebras don't get ulcers: The acclaimed guide to stress, stress-related diseases, and coping. New York, NY: Holt Paperbacks; 2004.

Disclosure: The authors have no disclosures. 

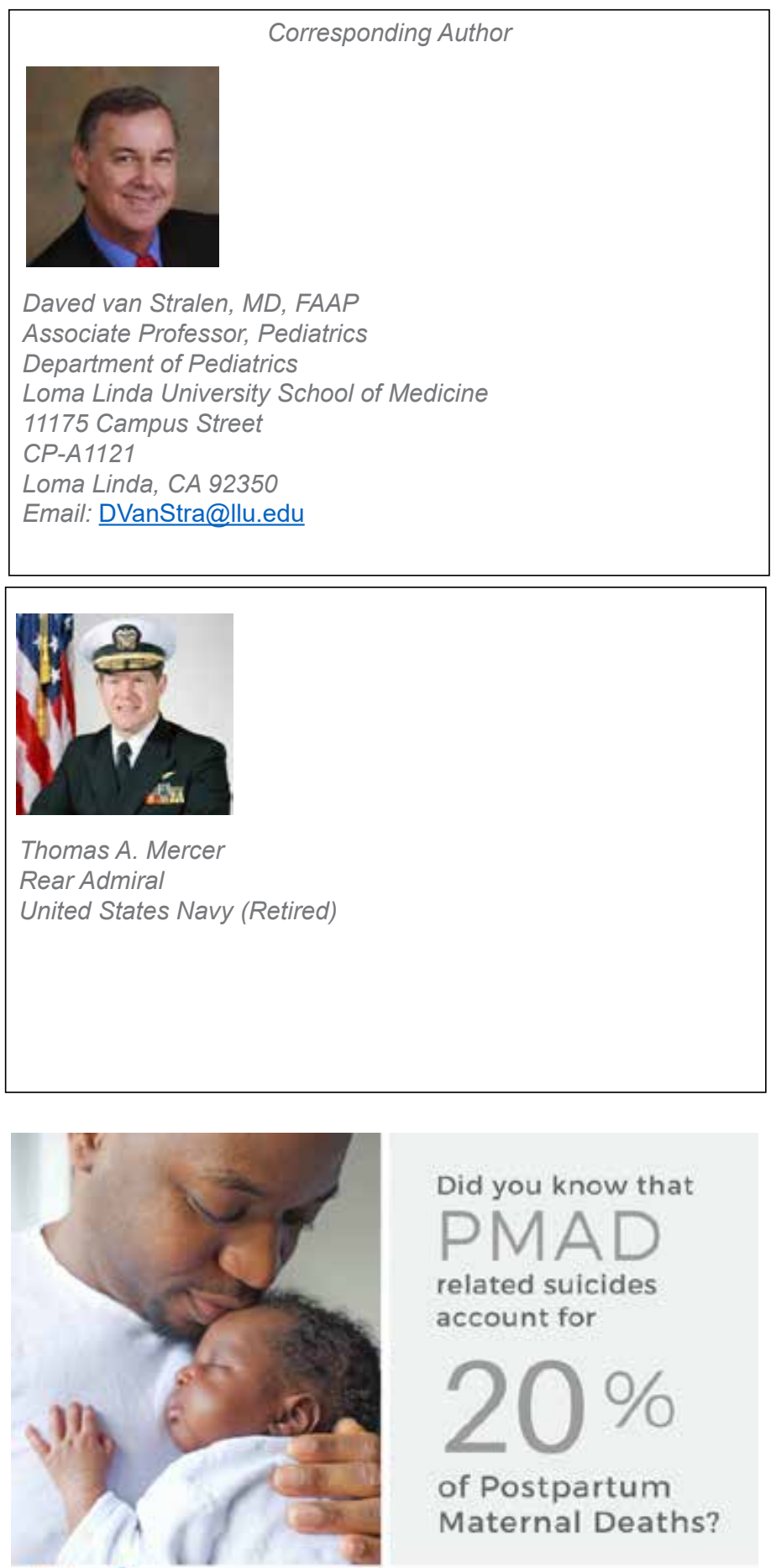

Did you know that

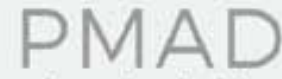

related suicides account for

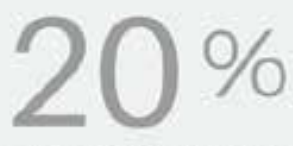

of Postpartum Maternal Deaths? Join O NPA

\section{Acknowledgments}

Raymond Novaco, Professor, Psychology and Social Behavior, School of Social Ecology, University of California, Irvine, California

Karl Weick- review and editing, Rensis Likert Distinguished University Professor of Organizational Behavior and Psychology, Emeritus, University of Michigan

William J. Corr, formerly with the Los Angeles City Fire Department, now deceased

Errol van Stralen, Ancora Education

Sean D. McKay, Element Rescue, LLC

William Gambino, CIV, DoD

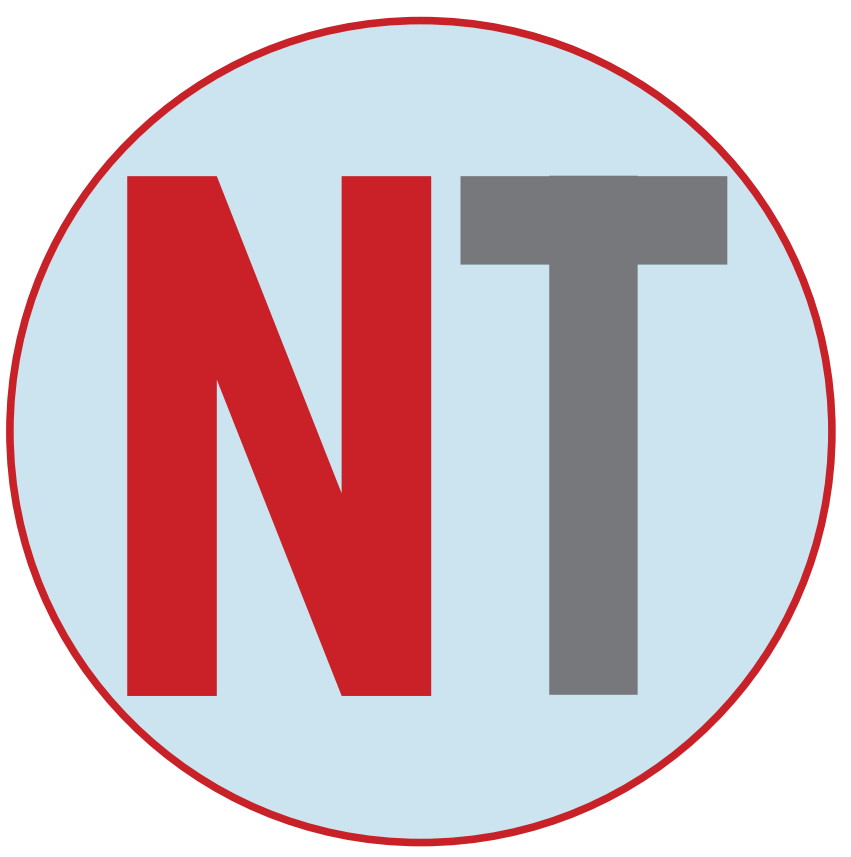

Readers can also follow NEONATOLOGY

via our Twitter Feed @NEOTO

\section{THE \\ BRETT TASHMAN} FOUNDATION
The Brett Tashman Foundation is a 501C(3) public charity. The mission of the Foundation is to find a cure for Desmoplastic Small Cell Round Tumors (DSRCT). DSRCT is an aggressive pediatric cancer for which there is no cure and no standard treatment. 100 percent of your gift will be used for research. There is no paid staff. To make your gift or for more information, go to "TheBrettTashmanFoundation.org" or phone (909) 981-1530. 